\title{
Nystatin enhances the immune response against Candida albicans and protects the ultrastructure of the vaginal epithelium in a rat model of vulvovaginal candidiasis
}

\author{
Xu Zhang ${ }^{1}$, Ting Li ${ }^{2}$, Xi Chen ${ }^{3}$, Suxia Wang ${ }^{1}$ and Zhaohui Liu ${ }^{2^{*}}$ (D)
}

\begin{abstract}
Background: Vulvovaginal candidiasis (WC) is a common infectious disease of the lower genital tract. Nystatin, a polyene fungicidal antibiotic, is used as a topical antifungal agent for WC treatment. The aim of the current study was to investigate the possible immunomodulatory effects of nystatin on the vaginal mucosal immune response during Candida albicans infection and examine its role in protection of vaginal epithelial cell (VEC) ultrastructure.

Results: Following infection with C. albicans, IFN- $\gamma$ and IL-17 levels in VECs were significantly elevated, while the presence of IgG was markedly decreased as compared to uninfected controls $(P<0.05)$. No significant differences in IL4 expression were observed. After treatment with nystatin, the level of IFN- $\gamma$, IL-17 and IgG was dramatically increased in comparison to the untreated group $(P<0.05)$. Transmission electron microscopy revealed that $C$. albicans invades the vaginal epithelium by both induced endocytosis and active penetration. Nystatin treatment protects the ultrastructure of the vaginal epithelium. Compared with the untreated C. albicans-infected group, Flameng scores which measure mitochondrial damage of VECs were markedly decreased $(P<0.001)$ and the number of adhesive and invasive $C$. albicans was significantly reduced $(P<0.01)$ after treatment with nystatin.

Conclusions: Nystatin plays a protective role in the host defense against $C$. albicans by up-regulating the IFN- $\gamma$-related cellular response, the IL-17 signaling pathway and possibly through enhancing VEC-derived IgG-mediated immunity. Furthermore, nystatin notably improves the ultramorphology of the vaginal mucosa, partially through the protection of mitochondria ultrastructure in VECs and inhibition of adhesion and invasion by C. albicans. Together, these effects enhance the immune response of the vaginal mucosa against $C$. albicans and protect the ultrastructure of vaginal epithelium in WC rats.
\end{abstract}

Keywords: Vulvovaginal candidiasis, Vaginal epithelial cells, Candida albicans, Nystatin, Cytokines

\section{Background}

Vulvovaginal candidiasis (VVC) is a common infectious disease of the lower genital tract, which affects approximately $75 \%$ of women of reproductive age. Among patients with VVC, 6-9\% suffer from recurrent VVC (RVVC) [1]. Most cases are caused by Candida albicans, one of the most common commensals, which acts as an opportunistic fungal pathogen in the

\footnotetext{
* Correspondence: 17301255426@163.com

²Department of Gynecology, Beijing Obstetrics and Gynecology Hospital,

Capital Medical University, Beijing 100026, China

Full list of author information is available at the end of the article
}

vagina. The interactions between $C$. albicans and host defense mechanisms play an important role in determining whether colonization remains harmless or leads to epithelium infection. C. albicans adheres to, invades and damages epithelial cells. Host defenses of the vaginal epithelium include a mechanical barrier against invading pathogens and the local innate immune response triggered by $C$. albicans infection $[2,3]$.

Previous studies [4-6] have identified putative roles for several immune mediators in local host defenses against microbial infection. It is speculated that recognition of Candida can activate an epithelial cell-mediated

(c) The Author(s). 2018 Open Access This article is distributed under the terms of the Creative Commons Attribution 4.0 International License (http://creativecommons.org/licenses/by/4.0/), which permits unrestricted use, distribution, and reproduction in any medium, provided you give appropriate credit to the original author(s) and the source, provide a link to the Creative Commons license, and indicate if changes were made. The Creative Commons Public Domain Dedication waiver (http://creativecommons.org/publicdomain/zero/1.0/) applies to the data made available in this article, unless otherwise stated. 
cytokine response, leading to the recruitment and activation of various immune cells including neutrophils, dendritic cells and $\mathrm{T}$ cells $[7,8]$. Interferon-gamma (IFN- $\gamma$ ) is a type II IFN produced by activated $\mathrm{T}$ cells, natural killer (NK) cells and natural killer $\mathrm{T}$ cells [9]. It activates phagocytes and favors the development of a Th1 protective response that participates in the clearance of fungal pathogens [10]. Expression of interleukin-4 (IL-4), the major cytokine involved in Th2 immune responses, correlates with disease exacerbation and pathology [11]. IL-17 has emerged as an essential mediator of protection against $C$. albicans in oral and dermal candidiasis [12]. It promotes antifungal immunity through up-regulation of pro-inflammatory cytokines, neutrophil-recruiting chemokines and antimicrobial peptides, which limit fungal overgrowth [13]. However, the role of IL-17-mediated immune responses in VVC is still controversial [14, 15]. In recent years, accumulating evidence has suggested that various non-B lineage cells including epithelial cells $[16,17]$ can produce immunoglobulin G (IgG). And the lymphoid-specific proteins RAG1 and RAG2, which are required for $\mathrm{V}(\mathrm{D}) \mathrm{J}$ recombination, are expressed in these cells. In addition, we previously showed that healthy vaginal epithelial cells (VECs) generated IgG in vitro [18]. However, the knowledge about the function of these non-lymphoid cell-derived Ig is still limited. Taken together, we speculate that Th2/Th1 balance may be related with antifungal activity against $C$. albicans.

Nystatin is an effective and broad-spectrum polyene fungicidal antibiotic which has been used for decades as a clinical antifungal agent for treating superficial candidiasis such as VVC $[19,20]$. It functions mainly through binding to ergosterol and forming barrel-like, membranespanning channels in the plasma membrane of antibioticsensitive organisms [21, 22]. Increased permeability of fungi leads to leakage of small molecules and disturbance of cellular electrochemical components and subsequently fungal death [23]. In addition, nystatin can sequester cholesterol located on the plasma membrane of eukaryotic cells and alter the micro-structure of lipid rafts [24], which has been shown to play crucial roles in regulating both innate and adaptive immune responses through pathogen recognition, lymphocyte activation and cytokine signaling [25]. Therefore, nystatin may influence host antimicrobial defense responses. Several studies have focused on the putative immunomodulatory effects of nystatin. It is reported that nystatin up-regulates human macrophages chemokine CCL2 and CXCL8 levels [26]. Additionally, nystatin has been shown to act as a Toll-like cell receptor agonist [27], which induces immune responses by recruiting immune cells and promoting the secretion of chemokines [28, 29]. Whether nystatin can regulate the expression of immune modulators to promote an anti-Candida vaginal immune response has not yet been fully investigated.
Transmission electron microscopy (TEM) is a standard tool to investigate interactions between pathogenic fungi and the host due to its high resolution. To the best of our knowledge, nystatin-associated ultrastructural changes to the vaginal epithelium during $C$. albicans infection have not been investigated. The aim of the present study was to evaluate the possible antifungal mechanisms and immunoregulatory role of nystatin in VVC and to assess the protective effects on vaginal mucosa from an ultrastructural perspective.

\section{Methods}

\section{Animals}

The present study was approved by the Animal Ethics Committee (AEC) of Peking University First Hospital (PUFH). Specific pathogen free, non-mated, female Sprague-Dawley (SD) rats weighing 180-200 g were purchased from the Animal Center of Peking University Health Science Center. SD rats were housed in cages with controlled temperature $\left(21 \pm 2{ }^{\circ} \mathrm{C}\right)$ and humidity $(30 \pm 5 \%)$, and a 12 / $12 \mathrm{~h}$ light/dark cycle. A standard laboratory diet and free access to tap water were supplied. After adaptation for 1 week, the animals were anesthetized with $30 \mathrm{mg} / \mathrm{kg}$ of phenobarbital sodium, ovariectomized and maintained in pseudo-estrus state by subcutaneous injection of estradiol hexa-hydrobenzoate, $0.5 \mathrm{mg} / \mathrm{week} / \mathrm{rat}$, administrated as fractions three times a week until the end of the experiments [30].

\section{Microbial strains}

C. albicans strains (ATCC-11006, provided by the Department of Dermatology laboratory, PUFH) were cultured aerobically on Sabouraud Dextrose Agar (SDA, Becton Dickinson, MD, USA) at $37{ }^{\circ} \mathrm{C}$ for $48 \mathrm{~h}$. One colony of fungal cells was collected and suspended in RPMI 1640 and adjusted to $5.0 \times 10^{8}$ yeasts $/ \mathrm{mL}$.

\section{Drug preparation}

Nystatin vaginal effervescent tablets (Sino-American Shanghai Squibb Pharmaceuticals, Ltd.) consisted of $1.0 \times 10^{5}$ units $(100 \mathrm{mg})$ of nystatin per tablet and other auxiliary components. One vaginal effervescent tablet was dissolved in $5 \mathrm{ml}$ of normal saline to prepare a drug solution of $2.0 \times 10^{4}$ units $/ \mathrm{mL}(20 \mathrm{mg} / \mathrm{mL})$ and stored at $-20{ }^{\circ} \mathrm{C}$ for further use.

\section{Establishment of a VVC model in rats}

Thirty SD rats were randomly divided into control $(n=$ $6)$ and experimental groups $(n=24)$. Rats in the experimental groups were injected vaginally with $0.1 \mathrm{~mL}$ of $C$. albicans suspension $\left(5.0 \times 10^{8}\right.$ yeasts $\left./ \mathrm{mL}\right)$ and the control rats were injected with the same volume of RPMI 1640. The opening to the vaginas was blocked with aseptic cotton balls to prevent the outflow of fluid. At day 4 
following inoculation, Gram staining of vaginal swabs from all rats was performed and examined by light microscopy (LM). Vaginal tissues biopsied from some of the infected rats were fixed in $4 \%$ paraformaldehyde, paraffin-embedded, sectioned and stained by hematoxylin-eosin (HE) to confirm inflammation. Rats with VVC were identified as those showing symptoms of inflammation and erythema, and having thick white vaginal discharges. Meanwhile, the presence of yeast and hyphae was confirmed by microscopy. Vaginal wash taken from each rat was cultured on SDA containing $50 \mathrm{mg} / \mathrm{mL}$ of chloramphenicol at $28{ }^{\circ} \mathrm{C}$ for $48 \mathrm{~h}$. The number of yeasts of each animal was counted and expressed as colony-forming units $(\mathrm{CFU}) / \mathrm{mL}$ at intervals during the vaginal infection.

After VVC confirmation, all rats in the experimental groups were randomly separated into nystatin-treated and untreated groups. To evaluate the time-dependent effects of C. albicans infection, rats in the untreated group were divided into $4 \mathrm{~d}, 8 \mathrm{~d}$ and $15 \mathrm{~d}$ sub-groups ( $n=6$ each group). Rats in treated group were injected vaginally with $2 \times 10^{4}$ units $/ \mathrm{mL}$ $(20 \mathrm{mg} / \mathrm{mL})$ of nystatin suspension for seven consecutive days. The dose of nystatin per day was determined according to animal equivalent dose calculations based on body surface area [31]. Meanwhile, the untreated rats received the same volume of normal saline vehicle (Fig. 1).

To determine drug efficacy of nystatin, the number of $C$. albicans (CFU) of vaginal wash was counted and the presence of hyphae or yeast of vaginal secretions was determined by Gram staining under LM in both nystatin-treated group and untreated $15 \mathrm{~d}$ sub-group. Samples with no detectable CFU together with no hyphae or yeast as determined by LM were defined as pathogen negative. The negative conversion rate (NCR) was calculated as the number of pathogen negative cases / $6 \times 100 \%$.

\section{Immunohistochemistry}

IFN- $\gamma$, IL-4, IL-17 and IgG levels were measured by immune labeling. Briefly, vaginal specimens were fixed in $4 \%$ paraformaldehyde, dehydrated in graded ethanol and embedded in paraffin. Deparaffinated sections $6 \mu \mathrm{m}$ thick were incubated with $3 \%$ hydrogen peroxide to eliminate endogenous peroxidase, blocked with bovine serum albumin (BSA, TBD Science Technology, Tianjin, China) at room temperature for $10 \mathrm{~min}$ and then incubated overnight at $4{ }^{\circ} \mathrm{C}$ with anti-rat-IFN- $\gamma$, anti-rat-IL-4, anti-ratIL-17 (rabbit polyclonal, Cloud-Clone, USA; 1:100 dilution for anti-rat-IFN- $\gamma$, 1:200 for anti-rat-IL-4 and 1:50 for anti-rat-IL-17) and anti-rat-IgG (RP125, provided by the Immunology Department, Peking University Health Science Center; 1:100 dilution), respectively. After rinsing with phosphate-buffered saline (PBS) three times, the sections were incubated with horseradish peroxidase conjugated anti-rabbit Ig (Zhongshan Golden Bridge Biotechnology). The negative control consisted of incubation with PBS instead of primary antibody. The results of immunohistochemistry were assessed by one experienced pathologist using a semi-quantitative analysis based on immunoreactivity score (IRS) [32]. The IRS-evaluation considers the percentage of stained area and visualized grade of color intensity by multiplying scores of staining percentage (SP; 0 , lower than $10 \% ; 1,10-25 \% ; 2,26-50 \%$; 3, 51-75\%; 4, 76-100\%) and staining intensity (SI; 0 , negative; 1 , mild; 2 , moderate; 3 , severe). The predominant grade of intensity was used as suggested previously [33] (Fig. 2).

\section{Transmission electron microscopy}

Fresh rat vaginal tissues were fixed in $3 \%$ glutaraldehyde for $3 \mathrm{~h}$ and $1 \%$ osmic acid for $1 \mathrm{~h}$ at $4{ }^{\circ} \mathrm{C}$. Following rinsing with $\mathrm{PBS}$ and dehydration in graded acetone, specimens were embedded in PON812 (SPI, West Chester, PA, USA) and polymerized at $60{ }^{\circ} \mathrm{C}$ for $24 \mathrm{~h}$.

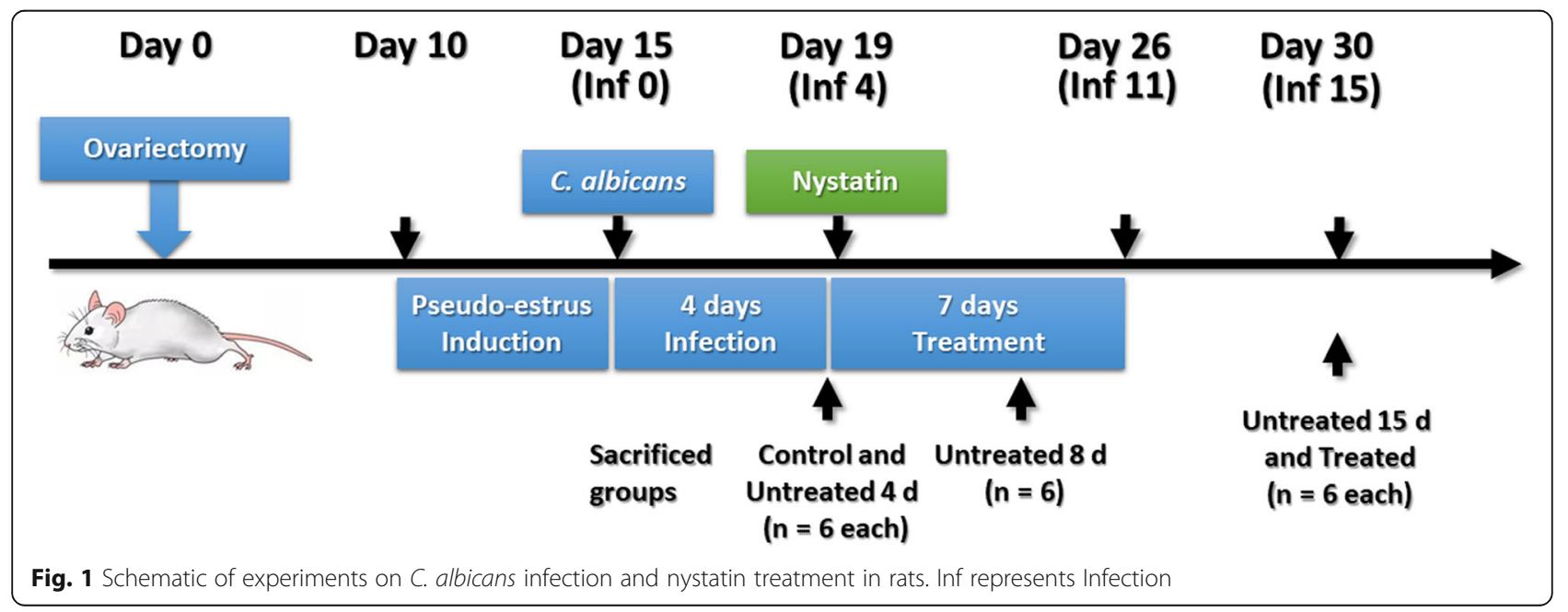




\section{Staining intensities (SI)}

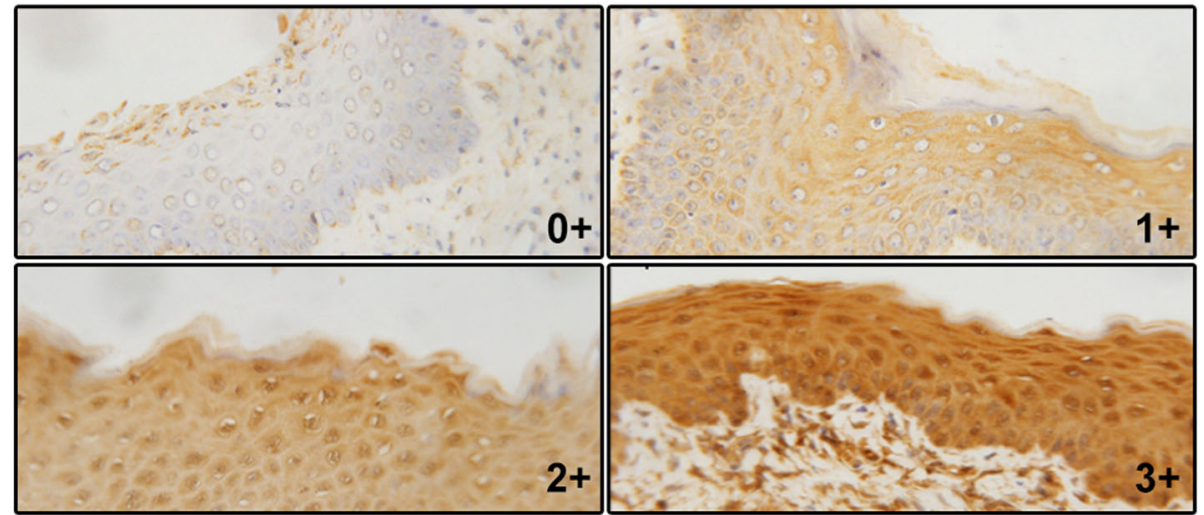

Fig. 2 Overview of the staining intensities of immunoreactivity score (IRS). The staining intensities were shown $(0+, 1+, 2+, 3+)$

Sections $70 \mathrm{~nm}$ thick were then double-stained with 5\% uranyl acetate and lead citrate. Ultrastructural changes in different groups were observed by JEM 1230 TEM (JEOL, Japan). Quantification of adhesive and invasive $C$. albicans, in both yeast and hyphal forms, in nystatin treated and untreated $15 \mathrm{~d}$ sub-groups, was performed under a JEOL 1200 EX in 10 consecutive fields. Scoring of mitochondria in VECs according to the Flameng grading system [34] was performed using a JEOL 1200 EX. A total of five fields were randomly chosen from each slice, with each field including 20 mitochondria for semi-quantitative analysis. Each mitochondrion was scored as follows: 0 , normal mitochondrial structure; 1 , normal mitochondrial structure but missing particles; 2 , mitochondrial swelling and transparent matrix without steep rupture; 3 , steep rupture and concentrated matrix; 4 , rupture, and incomplete inner and outer mitochondrial membranes.

\section{Statistical analysis}

Statistical analysis was performed using SPSS version 19.0 (SPSS Inc., Chicago, IL, USA). Comparison of NCRs was carried out by Fisher's exact test. All values are presented as the mean \pm standard deviation unless otherwise indicated. Comparisons were performed using an independent sample $t$ test, One-Way analysis of variance (ANOVA) and least significant difference (LSD) post hoc test. Calculated probabilities of 0.05 or less were considered to be statistically significant.

\section{Results}

\section{Nystatin effectively treats VVC}

The status of VVC in infected groups and its control rats were evaluated on $4 \mathrm{~d}$ post infection. Neither symptoms of VVC nor hyphae and yeast in the vaginal swabs were observed in the control rats. Meanwhile, cultures of vaginal wash were also negative. In contrast, all infected rats exhibited inflammation, swelling and vaginal discharges. The presence of yeast and hyphae and inflammation was determined under LM (Fig. 3a, b). Following an intravaginal inoculation with C. albicans, $>1 \times 10^{5}$ Candida $\mathrm{CFU} / \mathrm{mL}$ were detected $1 \mathrm{~h}$ post infection and the number slightly declined to $9 \times$ $10^{4} \mathrm{CFU} / \mathrm{mL} 4 \mathrm{~d}$ post infection (Fig. 3c). The successful rate of infection was $100 \%$.

To evaluate the antifungal effects of nystatin, we observed symptoms, microscopic findings and vaginal fungal loads in rats. For the untreated $15 \mathrm{~d}$ sub-group, inflammation, swelling and vaginal discharges, as well as positive vaginal swabs and cultures of vaginal wash persisted in all cases. The NCR was zero. After administration of nystatin, all rats in the treated group achieved complete symptomatic relief. No hyphae or yeast were observed by LM. Furthermore, compared with the untreated $15 \mathrm{~d}$ sub-group, nystatin treatment caused a significant decline in yeast vaginal counts on infection day $8(19.67 \pm 9.873$ vs $63.67 \pm 8.824, P<0.001)$ and $15(0.00$ \pm 0.000 vs $34.17 \pm 9.239, P<0.001$ ) (Fig. 3 c). The NCR reached $100 \%(P=0.001)$.

\section{Nystatin regulates the expression of cytokines in VECS}

To determine whether the expression of cytokines defined by the IRS differed in the uninfected controls, the infected nystatin-treated and untreated rats, immunohistochemistry was performed. Compared with the control group ( $4.17 \pm 2.563)$, the expression levels of IFN $-\gamma$ in the untreated group were significantly increased: $4 \mathrm{~d}$ sub-group $(8.00 \pm 0.000, \quad P=0.008), 8 \mathrm{~d}$ sub-group $(7.83 \pm 2.714, P=0.011)$ and $15 \mathrm{~d}$ sub-group $(8.17 \pm$ 2.563, $P=0.006)$. The IFN- $\gamma$ IRS in the treated group was significantly higher than that of the untreated 15 d sub-group $(11.33 \pm 1.633$ vs. $8.17 \pm 2.563, P=0.029)$. No significant differences were observed among the untreated groups (Fig. 4). 


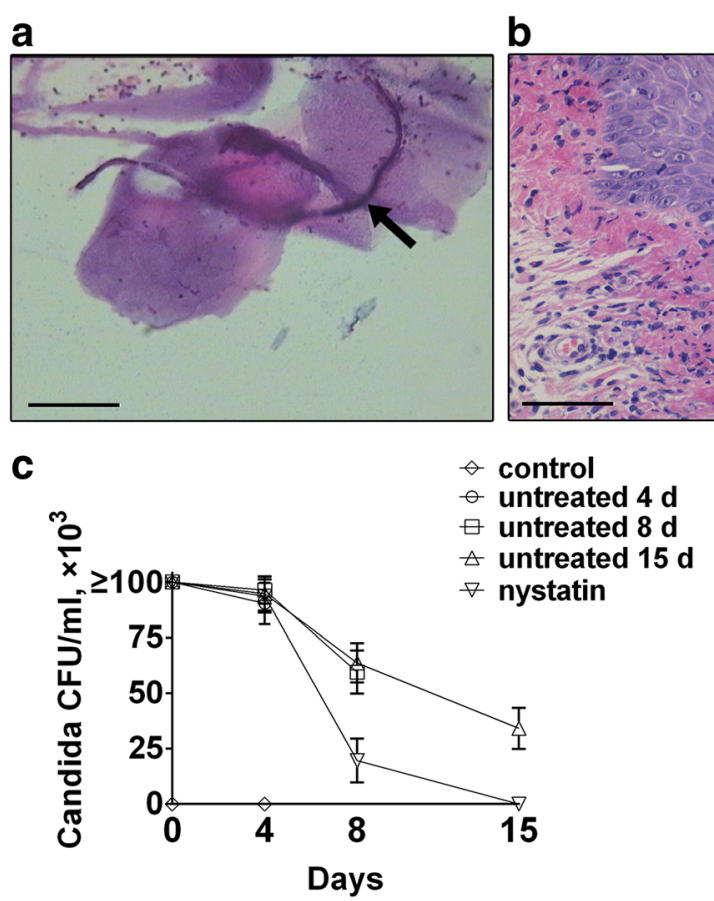

Fig. 3 Confirmation of WC model in rats and fungal burden from vaginal wash a, Light microscopy (LM) of Gram staining in a vaginal swab from rats infected by C. albicans. C. albicans hypha (arrow) adheres to vaginal epithelial cells. Scale bar $=20 \mu \mathrm{m}$, magnification $\times 1000$. b, Representative image of inflammation of infected vaginal epithelium by Hematoxylin-eosin (HE) staining. Neutrophils (arrow) infiltrate the epithelium. Scale bar = $50 \mu \mathrm{m}$, magnification $\times 400$. c, Outcome of vaginal fungal loads of rats infected with C. albicans $\left(5.0 \times 10^{8}\right.$ yeasts $\left./ \mathrm{mL}\right)$ in control, nystatin-treated and untreated $4 \mathrm{~d}, 8 \mathrm{~d}$ and $15 \mathrm{~d}$ sub-groups on days 0 ( $1 \mathrm{~h}$ post infection), $4 \mathrm{~d}, 8 \mathrm{~d}$ and $15 \mathrm{~d}$ post infection ( $\mathrm{n}=6 \mathrm{each}$ group). The vaginal $\mathrm{C}$. albicans colony-forming units (CFU) showed significant differences between in the nystatin-treated group and the untreated $15 \mathrm{~d}$ sub-group on both $8 \mathrm{~d}$ and $15 \mathrm{~d}$ post infection (All $P<0.001$ ).Data represent the mean \pm standard deviation

As shown in Fig. 4, no statistically significant differences were found in the levels of IL-4 expression among the different groups. However, compared with the control group $(8.67 \pm 1.633)$, the IL-4 IRS of the untreated groups decreased: $4 \mathrm{~d}$ sub-group $(7.17 \pm 2.041, P=$ $0.384), 8 \mathrm{~d}$ sub-group $(6.33 \pm 3.882, P=0.181)$ and $15 \mathrm{~d}$ sub-group $(8.17 \pm 3.488, P=0.770)$. After nystatin treatment, the expression of IL-4 exhibited an increasing trend (treated group vs. untreated $15 \mathrm{~d}$ sub-group, 10.67 \pm 2.066 vs. $8.17 \pm 3.488$, respectively, $P=0.162$ ). We determined the Th2/Th1 balance by calculating the IRS ratios of IL-4/IFN- $\gamma$ (Table 1). Compared with the control group, the Th2/Th1 IRS ratios in the untreated $4 \mathrm{~d}$ sub-group $(P=0.004), 8 \mathrm{~d}$ sub-group $(P=0.005)$ and $15 \mathrm{~d}$ sub-group $(P=0.009)$ were significantly decreased. No significant difference was detected between the untreated $15 \mathrm{~d}$ sub-group and nystatintreated group $(P=0.840)$.

The mean IRS for IL-17 in control rats was $4.67 \pm$ 2.066. After infection with C. albicans for four days, the expression of IL-17 was significantly up-regulated (8.67 $\pm 1.633, P=0.006)$. However, the IRS in the $8 \mathrm{~d}$ sub-group $(6.33 \pm 2.338, P=0.219)$ and $15 \mathrm{~d}$ sub-group $(6.33 \pm 2.875$, $P=0.219)$ showed no significant differences compared to control rats. Nystatin treatment stimulated a dramatic up-regulation in IL-17 compared with the untreated $15 \mathrm{~d}$ sub-group ( $10.67 \pm 2.066$ vs. $6.33 \pm 2.875$, respectively, $P=$ $0.013)$. There were no statistically significant differences in IL-17 levels across the untreated groups (Fig. 4).

\section{Nystatin enhances IgG expression in vaginal epithelial cells}

Compared with control rats $(10.05 \pm 2.510)$, the expression of IgG was significantly lower in the untreated groups: $4 \mathrm{~d}$ sub-group $(7.00 \pm 1.673, P=0.033), 8 \mathrm{~d}$ subgroup (5.83 $\pm 2.858, P=0.006)$ and $15 \mathrm{~d}$ sub-group (6.67 $\pm 3.266, P=0.021)$. However, nystatin administration significantly up-regulated the levels of IgG expressed by VECs as compared to the untreated $15 \mathrm{~d}$ sub-group (11.33 \pm 1.633 vs. $6.67 \pm 3.266$, respectively, $P=0.011)$. No significant differences were observed across the untreated groups (Fig. 4).

\section{Nystatin protects the infected vagina against $C$. albicans}

To further investigate the interactions between $C$. albicans and VECs, as well as host defense mechanisms and the protective effects of nystatin, we performed TEM to observe the adhesion and invasion of the fungal pathogen 


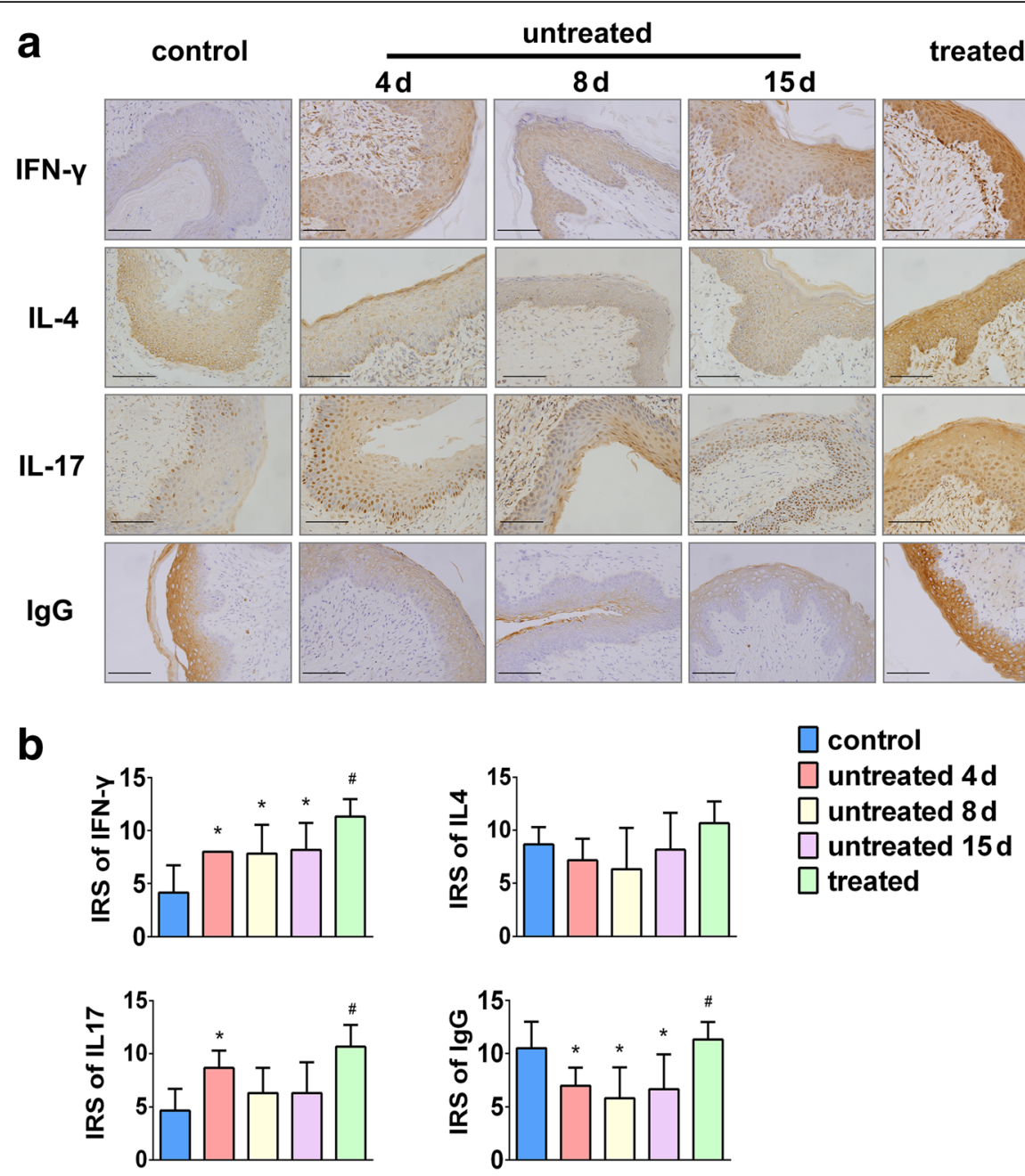

Fig. 4 Effects of nystatin on the expression of cytokines and IgG in VECs in response to C. albicans infection. Rats in the untreated group were infected for either 4, 8 or 15 d with C. albicans. a, Representative images of immunolabeling of IFN- $\gamma$, IL-4, IL-17 and IgG in control, untreated and nystatin treated groups. Scale bar $=50 \mu \mathrm{m}$, magnification $\times 400$. $\mathbf{b}$, Semi-quantitative analysis revealed healthy VECs express low levels of IFN- $\gamma$, IL4 and IL-17 and high level of IgG. A marked increase in the immunoreactivity score (IRS) of IFN- $\gamma$, IL-17 and a significant decrease of IgG were detected in untreated rats. After nystatin treatment, the IRS of IFN-y, IL-17 and IgG was significantly up-regulated. No statistically significant differences were found in the levels of IL-4 expression among the different groups. Data represent the mean \pm standard deviation $(n=6)$. * $P<0.05$ compared with control group. ${ }^{\#} P<0.05$ compared with the untreated 15 d sub-group

Table 1 IRS ratios of Th2/Th1 cytokines in control, untreated and nystatin-treated groups

\begin{tabular}{|c|c|c|}
\hline Group & Th2/Th1 IRS ratio & $P$ \\
\hline Control & $3.14 \pm 2.57$ & \\
\hline \multicolumn{3}{|c|}{ Untreated } \\
\hline $4 d$ & $0.90 \pm 0.26$ & $0.004^{a}$ \\
\hline $8 d$ & $0.94 \pm 0.70$ & $0.005^{a}$ \\
\hline $15 d$ & $1.12 \pm 0.62$ & $0.009^{a}$ \\
\hline Treated & $0.97 \pm 0.31$ & $0.840^{\mathrm{b}}$ \\
\hline
\end{tabular}

into the vaginal epithelium, as well as ultrastructural restoration in treated rats.

The vaginal mucosa in control rats consisted of nonkeratinized stratified squamous epithelium with desmosomes that bound adjacent cells together (Fig. 5a, b). The epithelial cells showed abundant tonofibrils and glycogen, and mitochondria with normal structures were distributed in the cytoplasm (Fig. 5b). Following infection with $C$. albicans, the vaginal mucosa of untreated rats exhibited ultrastructural impairment. In the early stage of infection, numerous neutrophils were recruited and infiltrated the vaginal epithelium and lamina propria (Fig. 5c). Superficial epithelial cells displayed a large number of lipid droplets, mucus granules and decreased glycogen deposits. The mitochondria in VECs were mildly or 


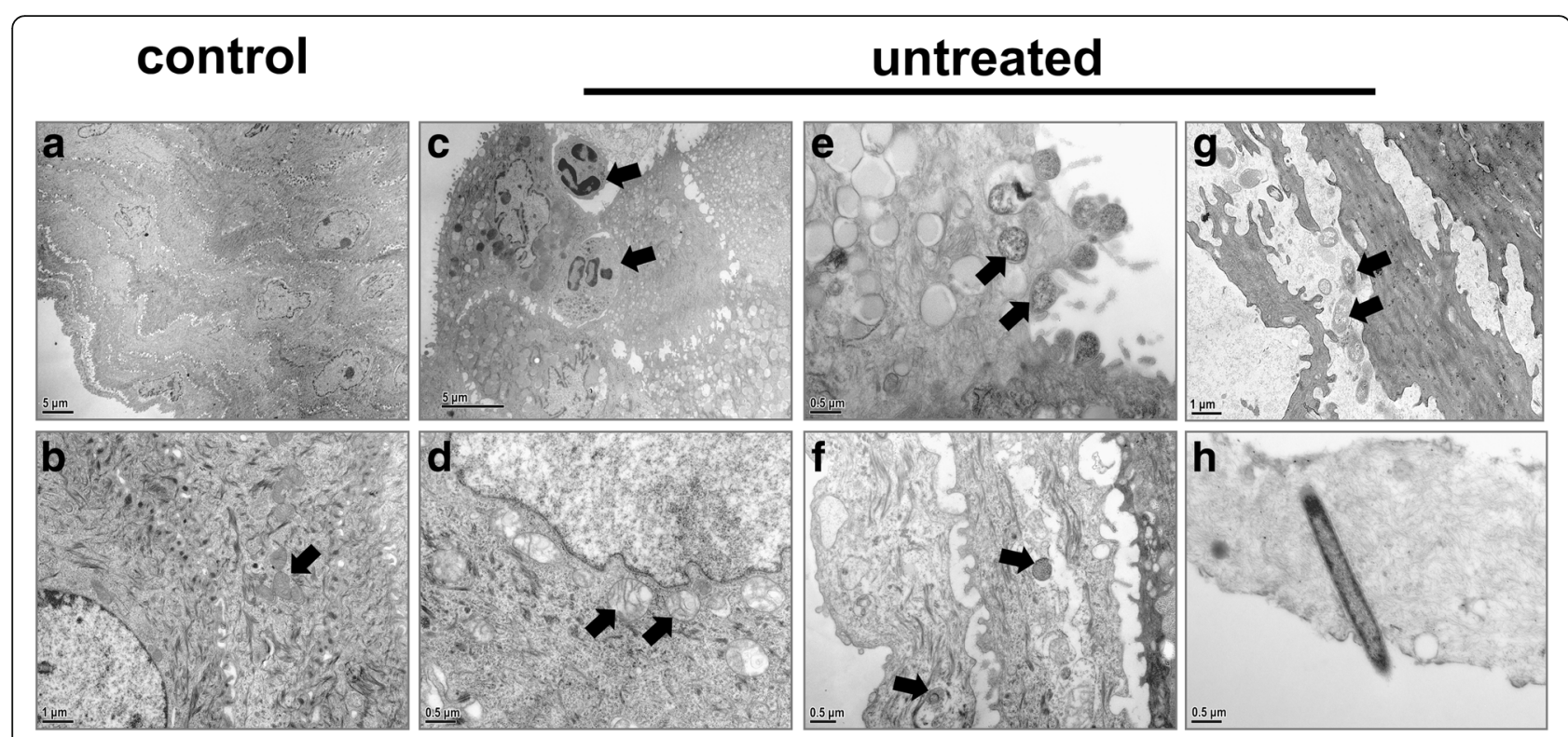

Fig. 5 Ultrastructure of the vaginal epithelium in the control group and untreated $4 \mathrm{~d}$ and $8 \mathrm{~d}$ sub-groups in the early stages of $C$. albicans infection. $\mathbf{a}$, Rats in the control group show normal vaginal morphology with a non-keratinized stratified squamous epithelium. Magnification $\times 3000$. $\mathbf{b}$, Adjacent VECs were bounded together by desmosomes and mitochondria (arrow) with normal morphology were observed in VECs from control rats. Magnification $\times 15,000$. c, A large number of neutrophils (arrow) infiltrate into the vaginal epithelium after C. albicans infection. Magnification $\times 6000$. d, Mitochondria (arrow) in VECs are mildly or moderately swollen with irregularly arranged cristae and decreased matrix density. Magnification $\times 30,000$. e, VEC pseudopods enveloped yeasts. Arrows indicate yeasts integrated with VECs. Magnification $\times 30,000$. $\mathbf{f}$, Yeasts (arrow) invaded into the superficial layers of the vaginal mucosa. Magnification $\times 25,000$. g, C. albicans hyphae (arrow) observed on the surface of the vaginal epithelium. Magnification $\times 15,000$. h, A hypha penetrated into fiber-like keratinized materials of the VEC surface. Magnification $\times 30,000$

moderately swollen with decreased matrix density (Fig. 5d). Elongated finger-like and/or bubble-like pseudopods protruded from the surface of VECs. The pseudopods contacted with C. albicans yeast cells and appeared to engulf these organisms (Fig. 5e). In addition, yeasts and hyphae penetrated superficial layers of the vaginal mucosa (Fig. $5 \mathrm{f}-5 \mathrm{~h})$. At infection day 15, the entire epithelial layer was destroyed (Fig. 6a). Epithelial cells exhibited a shrunken morphology characteristic of necrosis (Fig. 6b), resulting in damage to desmosomes and increased intercellular spaces (Fig. 6c). Mitochondria in VECs were markedly enlarged, with transparent matrix, ruptured cristae and incomplete inner and outer mitochondrial membranes (Fig. $6 \mathrm{~d})$. A greater number of yeasts were observed in the cytoplasm of VECs at day 15 compared to the early stage of infection (Fig. 6e). The yeasts are irregular shaped and seem to reproduce by budding (Fig. 6f). In the nystatin-treated group, the ultrastructure of the vaginal epithelium was notably improved (Fig. 7a). Few scattered inflammatory cells were observed in the entire layer of mucosa (Fig. 7b). The width of the intercellular spaces decreased and no damage was observed at cell junctions (Fig. 7c, d). The morphology of the mitochondria in VECs was normal or showed only slight swelling (Fig. 7e). Furthermore, compared with the untreated $15 \mathrm{~d}$ sub-group, nystatin treatment significantly reduced the number of adhesive and invasive C. albicans in both yeast and hyphal forms respectively (Table 2, Fig. 7f).

The ultrastructure of the mitochondria is regarded as an early morphological indicator of cell damage. Therefore, we determined the Flameng scores of mitochondria in VECs among different groups. As shown in Table 3, the Flameng scores of mitochondria in untreated $4 \mathrm{~d}, 8$ $\mathrm{d}$ and $15 \mathrm{~d}$ sub-groups were significantly higher as compared with the control group (all $P<0.001$ ). Furthermore, in untreated sub-groups, a significant increase in Flameng score was observed with prolonged $C$. albicans infection (all $P<0.001$ ). Administrated of nystatin significantly decreased the Flameng scores of mitochondria in VECs $(P<0.001)$.

\section{Discussion}

The vaginal mucosa serves as the first line of defense against $C$. albicans through its anatomical and physiological characteristics, including the physical barrier of the epithelium, the shedding and renewal of VECs, and the vaginal $\mathrm{pH}$. It has been shown that recognition of $C$. albicans by epithelial cells induces a series of cytokines, which is immensely important in innate immune defense and pathogen elimination [7, 35]. Our present study shows that healthy VECs express low levels of IFN- $\gamma$, IL-4 and IL-17. After C. albicans infection, expression of 

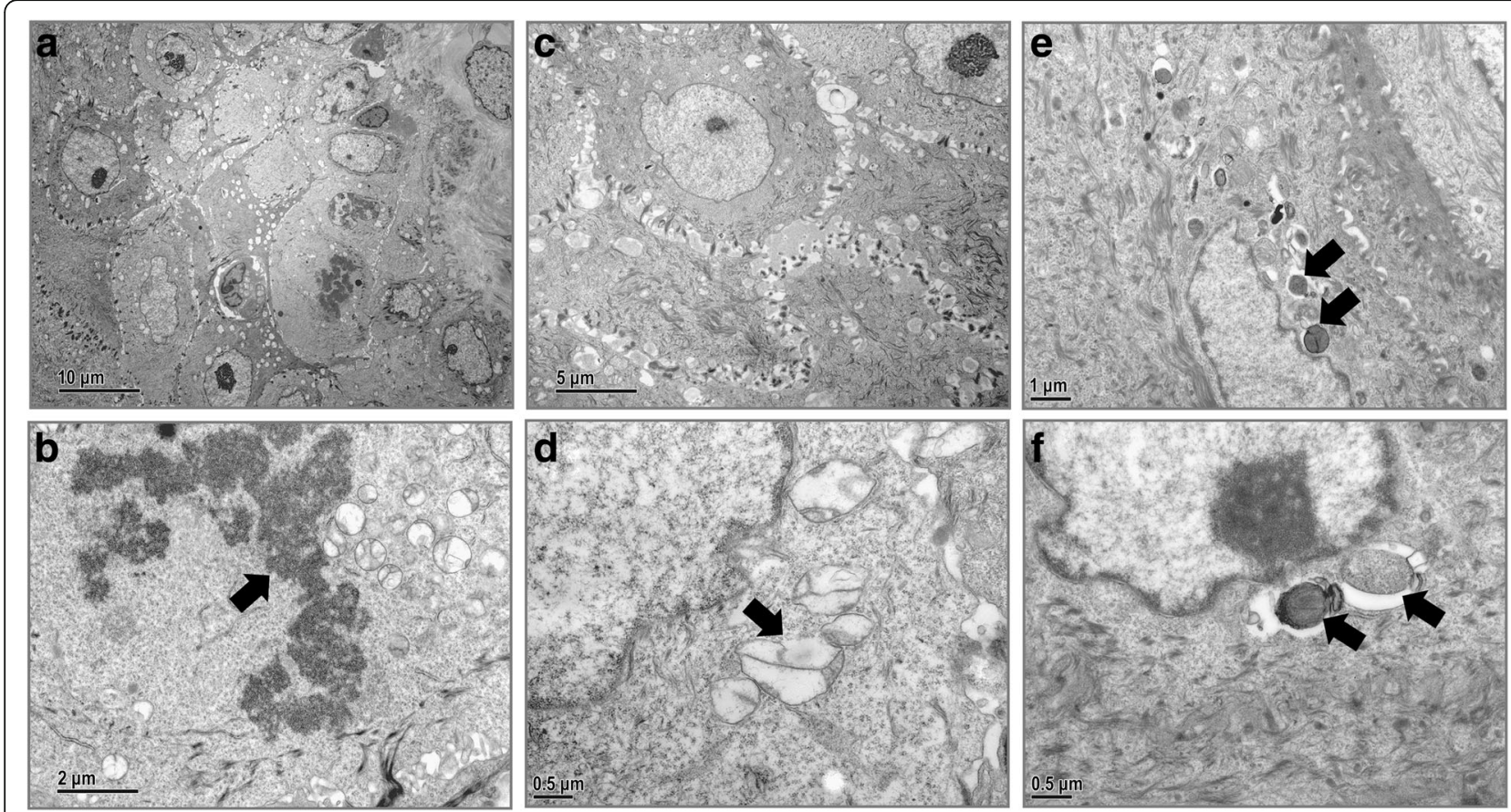

Fig. 6 Ultrastructural changes in the vaginal epithelium infected with C. albicans in the untreated $15 \mathrm{~d}$ sub-group. a, The vaginal mucosa is extensively damaged and inflammatory cells have infiltrated the vaginal epithelium. Magnification $\times 3000$. b. Karyolysis with dissolved chromatin (arrow) of the VECs. Magnification $\times 15,000$. c, Desmosomes between adjacent epithelial cells destroyed and intercellular spaces widened. Magnification $\times 6000$. d, Mitochondria in VECS significantly swollen with decreased matrix density and incomplete rupture of the inner and outer mitochondrial membranes (arrow). Magnification $\times 30,000$. e, More yeasts (arrow) are observed in the epithelial cytoplasm. Magnification $\times 15,000$. f, The yeasts (arrow) in VECs are of irregular shape and appear to reproduce by budding. Magnification $\times 30,000$

IFN- $\gamma$ and IL-17 increases. In addition, large number of neutrophils are observed in the epithelium, suggesting that activation and recruitment of immune cells into the mucosal layer might be involved in the pathogenesis of VVC. Elevated levels of IFN- $\gamma$ and IL-17 indicate that host immune defenses are activated in the early stages of C. albicans infection. Furthermore, we noted that IFN- $\gamma$ and IL-17 levels are remarkably increased with nystatin treatment. We speculate that a relatively high level of cytokines is a prerequisite for activating a protective mucosal response or maintaining homeostasis [36], whereas cytokine levels in untreated epithelium failed to further enhance the initiated immune processes. Therefore, we propose that nystatin could play an immunoregulatory role by increasing IFN- $\gamma$ and IL-17 levels to enhance vaginal anti-fungal immunity.

Previous studies $[37,38]$ have demonstrated that the balance of Th2/Th1 cytokines is involved in the pathogenesis of VVC and RVVC, determining susceptibility to C. albicans and the symbiotic relationship between the mucosa and pathogens. Generally, Th2 cytokines participate in the humoral immune response associated with enhanced vulnerability to pathogenic infection, while the Th1 cytokine response induces protective mucosal immunity. IL-17 is also essential for mucosal defense against C. albicans by recruitment of neutrophils and induction of antimicrobial peptides [39, 40]. Based on our results, in early stages of $C$. albicans infection, there is a significant decrease in the Th2/Th1 ratio and an increase in IL-17 in VECs. This suggests that the VEC-mediated local cellular immune response is enhanced and a rapid secretion of IL-17 by VECs is involved in protection against $C$. albicans. With infection time prolonged, it was noted that the ratio of Th2/Th1 on infection day 15 increased with tissue deterioration as observed ultrastructurally, suggesting a decreased clearance of mucosal $C$. albicans with suppressed immunity. However, whether chronic infection after $15 \mathrm{~d}$ would bring the ratio back to uninfected levels still need to be confirmed. In contrast to the untreated group, nystatin treatment again shifts balance to Th1 with a comparable Th2/Th1 ratio and elevated IL-17 level. These findings suggest that nystatin might provide a protective stimulation by up-regulating the expression of Th1 cytokines and promote a Th17biased mucosal immune response to eliminate vaginal $C$. albicans.

IgG plays a vital role in the humoral immune response through antigen recognition, complement binding and activation of effector cells. It has traditionally been believed that the production of Ig molecules is restricted 

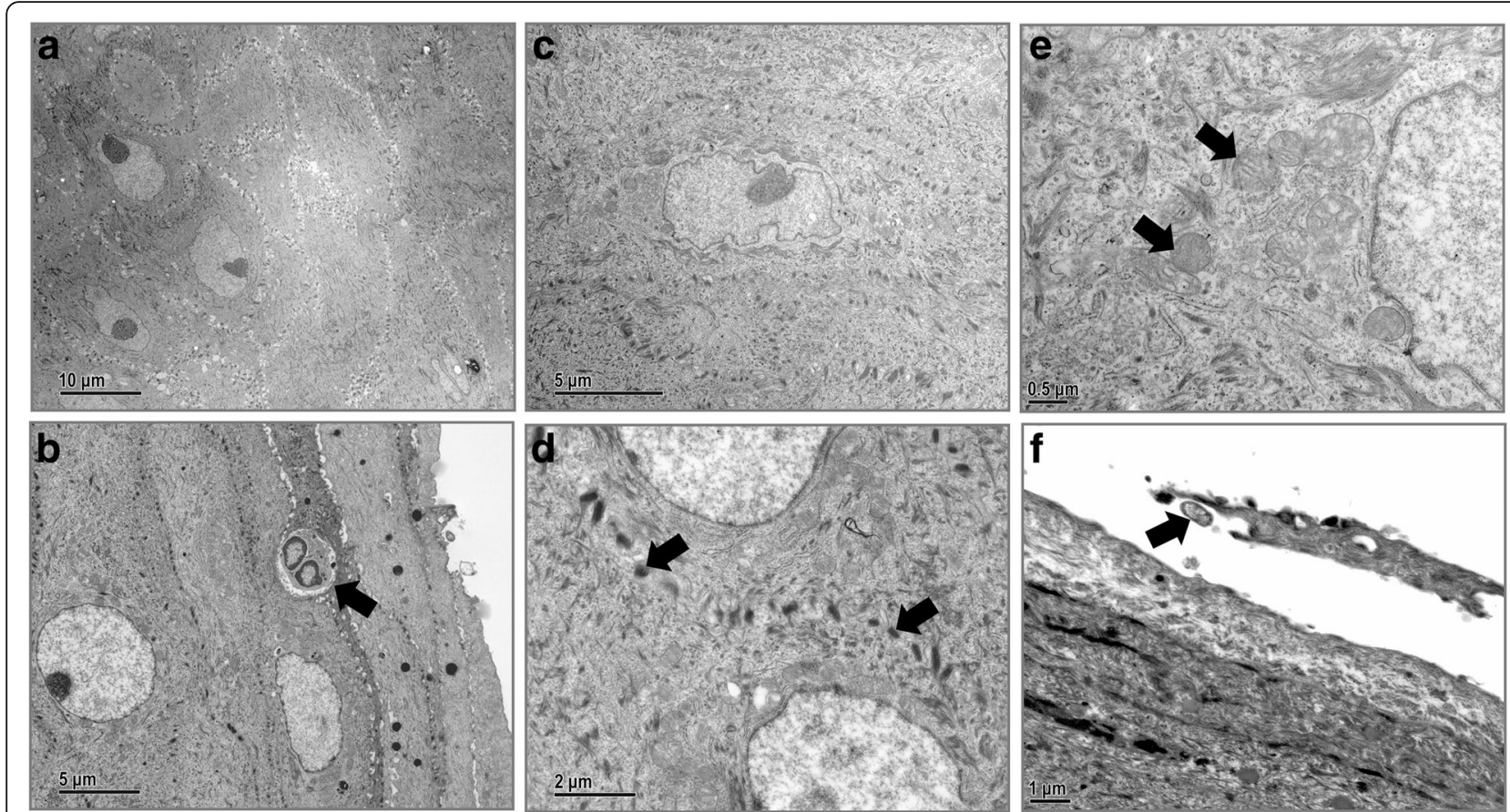

Fig. 7 Nystatin protects the ultrastructure of VECs from C. albicans infection. a, The vaginal epithelium was restored after nystatin treatment. Magnification $\times 3000$. b. Scattered neutrophils (arrow) observed in superficial VECs. Magnification $\times 6000$. c, The intercellular space between VECs is restored. Magnification $\times 8000$. d, The morphology of VEC junctions (arrow) is restored. Magnification $\times 15,000$. e, The ultrastructure of mitochondria (arrow) in VECs is restored to normal or showed only mild swelling. Magnification $\times 30,000$. f, Few hyphae (arrow) are detected on the surface of VECs. Magnification $\times 15,000$

to B lineage cells. However, Ig genes and proteins have been recently found in a variety of types of cells, including proliferating epithelial cells $[16,17]$. The Ig molecules expressed by these cells consist predominantly of IgG, and the light chains expressed are mainly kappa chains. Additionally, the functions of epithelial cell-origin Ig molecules are still unknown. In the present study, normal rat vaginal epithelium expressed a high level of IgG. The presence of IgG decreased following infection with C. albicans but was significantly up-regulated following nystatin treatment. Although, it is likely that VEC-derived IgG may be involved in local immune response, the antifungal role of non-lymphoid-origin IgGs is not unequivocally established yet. Further investigations are needed to confirm whether

Table 2 Quantification of adhesive and invasive C. albicans in nystatin-treated and untreated groups

\begin{tabular}{lllll}
\hline & Untreated & Treated & $t$ & $P$ \\
\hline Adhesive yeasts & $2.50 \pm 1.049$ & $0.00 \pm 0.000$ & 5.839 & 0.002 \\
Adhesive hyphae & $9.50 \pm 1.049$ & $1.50 \pm 0.548$ & 16.562 & $<0.001$ \\
Invasive yeasts & $12.33 \pm 1.966$ & $2.50 \pm 1.049$ & 10.808 & $<0.001$ \\
Invasive hyphae & $1.33 \pm 0.516$ & $0.00 \pm 0.000$ & 6.325 & 0.001
\end{tabular}

The number of adhesive and invasive C. albicans in the nystatin-treated group and untreated $15 \mathrm{~d}$ sub-group was evaluated by counting 10 consecutive fields under transmission electron microscopy (magnification $\times 1200$ ). Data represent the mean \pm standard deviation $(n=6)$
VEC-derived IgG participates in the antifungal immunity and the protective mechanisms of nystatin.

Mitochondria are involved in cellular events such as metabolism, bioenergetics, cell death, and innate immune signaling [41]. Fungal infections are associated with alterations in cellular physiology, which directly or indirectly impairs mitochondrial ultramorphology. An accumulation of injured mitochondria activates a vicious cycle of mitochondrial damage and cell death [42]. Generally, C. albicans-induced VEC damage is characterized by mitochondrial swelling [36]. In the current study, our

Table 3 Flameng scores of mitochondria in VECs in control, untreated and nystatin treated groups

\begin{tabular}{lll}
\hline Group & Flameng score & $P$ \\
\hline Control & $0.13 \pm 0.342$ & \\
Untreated & & \\
$4 d$ & $0.72 \pm 0.636$ & $P<0.001^{\mathrm{a}}$ \\
$8 \mathrm{~d}$ & $1.94 \pm 0.904$ & $P<0.001^{\mathrm{a}, \mathrm{b}}$ \\
$15 \mathrm{~d}$ & $3.10 \pm 0.542$ & $P<0.001^{\mathrm{a}, \mathrm{b}, \mathrm{c}}$ \\
Treated & $0.51 \pm 0.707$ & $P<0.001^{\mathrm{d}}$ \\
\hline
\end{tabular}

Flameng scores of mitochondria in VECs were assessed by transmission electron microscopy for different groups. ${ }^{a} P<0.001$ compared with control group. ${ }^{b} P<0.001$ compared with untreated 4 d sub-group. ${ }^{c} P<0.001$ compared with untreated $8 \mathrm{~d}$ sub-group. ${ }^{\mathrm{d}} P<0.001$ compared with untreated $15 \mathrm{~d}$ sub-group. Data represent the mean \pm standard deviation $(n=6)$ 
ultrastructural findings provide evidence that $C$. albicans invades VECs through induced endocytosis and active penetration, which is consistent with our previous in vitro observations by scanning electron microscopy [18]. In our study, a prolonged infection time resulted in deterioration of the ultrastructure of the vaginal mucosa in untreated rats and altered morphology of mitochondria in VECs. However, with administration of nystatin, we observed a significant decrease in both intra- and extracellular $C$. albicans. Furthermore, the ultrastructure of the vaginal epithelium and mitochondria in VECs was significantly improved. These ultrastructural observations indicate that the protective anti-fungal effects of nystatin on the vaginal mucosa in VVC rats are partially associated with its capacity to protect mitochondrial morphology and inhibit adhesion and invasion by the fungal pathogen.

\section{Conclusions}

Our current study indicates that nystatin plays a protective role in the host defense against $C$. albicans by: (1) activating functional cytokines through up-regulation of IFN- $\gamma$ and IL-17 signaling; (2) significantly increasing the expression of VEC-derived IgG and likely modulating the antibody-mediated pathway; (3) exerting fungicidal effects by inhibiting adhesion and invasion of C. albicans; and (4) shielding the ultrastructure of the vaginal epithelium partially through protecting the morphology of mitochondria in VECs. Further studies are needed to investigate the molecular mechanisms of nystatin-mediated regulation of the vaginal epithelial immune response during VVC in more detail.

\section{Abbreviations \\ AEC: Animal Ethics Committee; ANOVA: One-Way analysis of variance; BSA: Bovine serum albumin; C. albicans: Candida albicans; CFU: Colony- forming units; HE: Hematoxylin-eosin; IFN-y: Interferon-gamma; IgG: Immunoglobulin G; IL-17: Interleukin-17; IL-4: Interleukin-4; IRS: Immunoreactivity score; LM: Light microscopy; LSD: Least significant difference; NCR: Negative conversion rate; NK: Natural killer; PBS: Phosphate-buffered saline; PUFH: Peking University First Hospital; RVVC: Recurrent vulvovaginal candidiasis; SD: Sprague-Dawley; SDA: Sabouraud Dextrose Agar; SI: Staining intensity; SP: Staining percentage; TEM: Transmission electron microscopy; VECs: Vaginal epithelial cells; WC: Vulvovaginal candidiasis}

\section{Acknowledgements}

Not applicable.

\section{Funding}

This work was supported by a grant from the National Natural Science Foundation of China [Grant No. 81571394]. The funding body did not have any influence in the design of the study or the collection, analysis, and interpretation of data as well as in writing the manuscript.

\section{Availability of data and materials}

All the data supporting our findings is contained within the manuscript.

\section{Authors' contributions}

$X Z, X C$ and $Z L$ conceived and designed the study; $X Z$ and $X C$ performed the experiments; XZ and SW analyzed data; XZ drafted the manuscript. ZL and $T L$ revised the manuscript. All authors read and approved the final manuscript.

\section{Ethics approval}

The animal work presented in this study was approved by Animal Center of Peking University Health Science Center and the Animal Ethics Committee (AEC) of Peking University First Hospital (201612003).

\section{Consent for publication}

Not applicable.

\section{Competing interests}

The authors declare that they have no competing interests.

\section{Publisher's Note}

Springer Nature remains neutral with regard to jurisdictional claims in published maps and institutional affiliations.

\section{Author details}

'Laboratory of Electron Microscopy, Ultrastructural Pathology Center, Peking University First Hospital, Beijing 100034, China. ${ }^{2}$ Department of Gynecology, Beijing Obstetrics and Gynecology Hospital, Capital Medical University, Beijing 100026, China. ${ }^{3}$ Department of Gynecology Minimally Invasive Center, Beijing Obstetrics and Gynecology Hospital, Capital Medical University, Beijing 100026, China.

Received: 19 February 2018 Accepted: 15 October 2018

Published online: 25 October 2018

References

1. Sobel JD. Recurrent vulvovaginal candidiasis. Am J Obstet Gynecol. 2016; 214:15-21.

2. Naglik JR, Moyes D. Epithelial cell innate response to Candida albicans. Adv Dent Res. 2011;23:50-5.

3. Duhring S, Germerodt S, Skerka C, Zipfel PF, Dandekar T, Schuster S. Hostpathogen interactions between the human innate immune system and Candida albicans-understanding and modeling defense and evasion strategies. Front Microbiol. 2015;6:625

4. Borish LC, Steinke JW. 2. Cytokines and chemokines. J Allergy Clin Immunol. 2003;111:S460-75.

5. Basu S, Quilici C, Zhang HH, Grail D, Dunn AR. Mice lacking both G-CSF and IL-6 are more susceptible to Candida albicans infection: critical role of neutrophils in defense against Candida albicans. Growth Factors. 2008; 26:23-34.

6. Onishi RM, Gaffen SL. Interleukin-17 and its target genes: mechanisms of interleukin-17 function in disease. Immunology. 2010;129:311-21.

7. Moyes DL, Naglik JR. Mucosal immunity and Candida albicans infection. Clin Dev Immunol. 2011. https://doi.org/10.1155/2011/346307.

8. Weindl G, Naglik JR, Kaesler S, Biedermann T, Hube B, Korting HC, Schaller M. Human epithelial cells establish direct antifungal defense through TLR4mediated signaling. J Clin Invest. 2007;117:3664-72.

9. Qin $Y$, Zhang C. The regulatory role of IFN-gamma on the proliferation and differentiation of hematopoietic stem and progenitor cells. Stem Cell Rev. 2017;13:705-12.

10. Gozalbo D, Maneu V, Gil ML. Role of IFN-gamma in immune responses to Candida albicans infections. Front Biosci (Landmark Ed). 2014;19:1279-90.

11. Altamura M, Casale D, Pepe M, Tafaro A. Immune responses to fungal infections and therapeutic implications. Curr Drug Targets Immune Endocr Metabol Disord. 2001;1:189-97.

12. Conti HR, Gaffen SL. IL-17-mediated immunity to the opportunistic fungal pathogen Candida albicans. J Immunol. 2015;195:780-8.

13. Sparber F, LeibundGut-Landmann S. Interleukin 17-mediated host defense against Candida albicans. Pathogens. 2015;4:606-19.

14. Pietrella D, Rachini A, Pines M, Pandey N, Mosci P, Bistoni F, d'Enfert C, Vecchiarelli A. Th17 cells and IL-17 in protective immunity to vaginal candidiasis. PLoS One. 2011;6:e22770.

15. Yano J, Kolls JK, Happel Kl, Wormley F, Wozniak KL, Fidel PL, Jr. The acute neutrophil response mediated by $\mathrm{S} 100$ alarmins during vaginal Candida infections is independent of the Th17-pathway. PLoS One 2012;7:e46311.

16. Niu N, Zhang J, Huang T, Sun Y, Chen Z, Yi W, Korteweg C, Wang J, Gu J. lgG expression in human colorectal cancer and its relationship to cancer cell behaviors. PLoS One. 2012;7:e47362. 
17. Peng J, Wang HC, Liu Y, Jiang JH, Lv WQ, Yang Y, Li CY, Qiu XY. Involvement of non-B cell-derived immunoglobulin $\mathrm{G}$ in the metastasis and prognosis of salivary adenoid cystic carcinoma. Oncol Lett. 2017;14:4491-8.

18. Li T, Niu X, Zhang X, Wang S, Liu Z. Recombinant human IFNalpha-2b response promotes vaginal epithelial cells defense against Candida albicans. Front Microbiol. 2017:8:697.

19. Ng AW, Wasan KM, Lopez-Berestein G. Development of liposomal polyene antibiotics: an historical perspective. J Pharm Pharm Sci. 2003;6:67-83.

20. Mendling W, Brasch J, Cornely OA, Effendy I, Friese K, Ginter-Hanselmayer G, Hof H, Mayser P, Mylonas I, Ruhnke M, et al. Guideline: vulvovaginal candidosis (AWMF 015/072), S2k (excluding chronic mucocutaneous candidosis). Mycoses. 2015;58(Suppl 1):1-15.

21. Coutinho A, Prieto M. Cooperative partition model of nystatin interaction with phospholipid vesicles. Biophys J. 2003;84:3061-78.

22. Silva L, Coutinho A, Fedorov A, Prieto M. Competitive binding of cholestero and ergosterol to the polyene antibiotic nystatin. A fluorescence study. Biophys J. 2006;90:3625-31.

23. Fujii G, Chang JE, Coley T, Steere B. The formation of amphotericin B ion channels in lipid bilayers. Biochemistry. 1997;36:4959-68.

24. Park H, Go YM, St John PL, Maland MC, Lisanti MP, Abrahamson DR, Jo H. Plasma membrane cholesterol is a key molecule in shear stressdependent activation of extracellular signal-regulated kinase. J Biol Chem. 1998;273:32304-11.

25. Varshney $P$, Yadav V, Saini N. Lipid rafts in immune signalling: current progress and future perspective. Immunology. 2016;149:13-24.

26. Kim DH, Rhim BY, Eo SK, Kim K. Differential regulation of CC chemokine ligand 2 and CXCL8 by antifungal agent nystatin in macrophages. Biochem Biophys Res Commun. 2013;437:392-6.

27. Salyer AC, Caruso G, Khetani KK, Fox LM, Malladi SS, David SA. Identification of Adjuvantic activity of amphotericin B in a novel, multiplexed, poly-TLR/ NLR high-throughput screen. PLoS One. 2016;11:e0149848.

28. Sharifi L, Mohsenzadegan M, Aghamohammadi A, Rezaei N, Tofighi Zavareh F, Bokaie S, Moshiri M, Azizi G, Mirshafiey A, Aghazadeh Z. Immunomodulatory effect of G2013 (a-L-Guluronic acid) on theTLR2 and TLR4 in human mononuclear cells. Curr Drug Discov Technol. 2018; 15:123-31.

29. Tyurin YA, Shamsutdinov AF, Kalinin NN, Sharifullina AA, Reshetnikova ID. Association of Toll-like Cell Receptors TLR2 (p.Arg753GLN) and TLR4 (p. Asp299GLY) polymorphisms with indicators of general and local immunity in patients with atopic dermatitis. J Immunol Res. 2017. https://doi.org/10. 1155/2017/8493545.

30. Carrara MA, Donatti L, Damke E, Svidizinski TI, Consolaro ME, Batista MR. A new model of vaginal infection by Candida albicans in rats. Mycopathologia. 2010;170:331-8.

31. Nair AB, Jacob S. A simple practice guide for dose conversion between animals and human. J Basic Clin Pharm. 2016;7:27-31.

32. Dereci O, Akgun S, Celasun B, Ozturk A, Gunhan O. Histological evaluation of the possible transformation of peripheral giant cell granuloma and peripheral ossifying fibroma: a preliminary study. Indian J Pathol Microbiol. 2017;60:15-20.

33. Remmele W, Stegner HE. Recommendation for uniform definition of an immunoreactive score (IRS) for immunohistochemical estrogen receptor detection (ER-ICA) in breast cancer tissue. Pathologe. 1987;8:138-40.

34. Flameng W, Borgers M, Daenen W, Stalpaert G. Ultrastructural and cytochemical correlates of myocardial protection by cardiac hypothermia in man. J Thorac Cardiovasc Surg. 1980;79:413-24.

35. Diamond G, Beckloff N, Ryan LK. Host defense peptides in the oral cavity and the lung: similarities and differences. J Dent Res. 2008;87:915-27.

36. Naglik JR, Moyes DL, Wachtler B, Hube B. Candida albicans interactions with epithelial cells and mucosal immunity. Microbes Infect. 2011;13:963-76.

37. Barousse MM, Van Der Pol BJ, Fortenberry D, Orr D, Fidel PL Jr. Vaginal yeast colonisation, prevalence of vaginitis, and associated local immunity in adolescents. Sex Transm Infect. 2004;80:48-53.

38. Ouyang W, Chen S, Liu Z, Wu Y, Li J. Local Th1/Th2 cytokine expression in experimental murine vaginal candidiasis. J Huazhong Univ Sci Technolog Med Sci. 2008;28:352-5.

39. Goupil M, Cousineau-Cote V, Aumont F, Senechal S, Gaboury L, Hanna Z, Jolicoeur P, de Repentigny L. Defective IL-17- and IL-22-dependent mucosal host response to Candida albicans determines susceptibility to oral candidiasis in mice expressing the HIV-1 transgene. BMC Immunol. 2014;15:49.
40. Hernandez-Santos N, Gaffen SL. Th17 cells in immunity to Candida albicans. Cell Host Microbe. 2012;11:425-35.

41. Weinberg SE, Sena LA, Chandel NS. Mitochondria in the regulation of innate and adaptive immunity. Immunity. 2015;42:406-17.

42. Kim SJ, Ahn DG, Syed GH, Siddiqui A. The essential role of mitochondrial dynamics in antiviral immunity. Mitochondrion. 2018:41:21-7.

\section{Ready to submit your research? Choose BMC and benefit from:}

- fast, convenient online submission

- thorough peer review by experienced researchers in your field

- rapid publication on acceptance

- support for research data, including large and complex data types

- gold Open Access which fosters wider collaboration and increased citations

- maximum visibility for your research: over $100 \mathrm{M}$ website views per year

At $\mathrm{BMC}$, research is always in progress.

Learn more biomedcentral.com/submissions 